\title{
Study on inhibitive action of bone marrow stromal cells transplantation on immunologic reaction of experimental allergic neuritis
}

\author{
Yanhong Yin ${ }^{1}$, Guozhong $\mathrm{Li}^{1}$ \\ ${ }^{1}$ Department of Neurology, the first Affiliated Clinical Teaching College, Harbin Medical University, China
}

The study was designed to establish animal experimental groundworks for a clinical practicable therapeutic route of stem cell repair for Guillain-Barré syndrome from eradicating its imunopathology angle. We separated, cultivated, identified bone marrow derived stroma cells of healthy Lewis rat to obtain activatory specific $T$ lymphocyte by $P 0$ 180-199 compositus immunoemulsion induction in post foot pad of Lewis rat, thereby to set up P0 180-199 specific $\mathrm{T}$ cell line in vitro. CFA injection was used as contrast control. We set up experimental allergic neuritis model by P0180-199 specific $T$ lymphocytes adoptive immunity andset up allogeneil graft group by BMSCs injection from vena caudalis. PBS injection group was used as contrast control. Both groups were grade by EAN clinical score on 1 6 days after immune. All experimental animals were killed on 6 days after immune to observe lymphocyte infiltration status in sciatic nerve. $T$ lymphocyte proliferation test in vitro was done by separating, cultivating the obtained allergic T lymphocytes from both groups and stimulating them by P0180-199 or ConA, detecting their proliferation ability by $\beta$-counter. None antigen stimulation group was used as contrast control. At last we detected serum IFN- $\gamma$, TNF- $\alpha$ and IL-4 level of BMSC transplantation group and control group on 6 days after immune by ELISA method. The results shown BMSCs grown well when primary cultivated in vitro, shown stable function and strong cloning ability in serial subcultivation. BMSCs shown CD14 negative expression and CD44 positive expression, the cell purity was above $85 \%$. The sensitized process was slow and clinic symptoms were severe after immune from metapodium pad, obvious symptoms were observed on 10 days. Specific $\mathrm{T}$ cell line could be obtained by this method. Symptoms of adoptive immune animals were slight and appeared rapidly which were easy for experimental observation. There was significant difference in BMSCs transplantation and PBS control group by $P<0.001$ on $4 \sim 6$ days after adoptive immune; The infiltration cells was found much more in control group than in BMSC group by $P<0.01$. Specific P0 antigen or ConA stimulation test shown: Lymphocytes shown lower proliferation ability in BMSCs transplantation group than in PBS control group by $\boldsymbol{P}<0.01$, the difference was significant; There are no significant difference in both group without any antigen stimulation. Serum IFN- $\gamma$ level was higher in control group than in BMSC group by $P<0.01$; TNF- $\alpha$ level was obviously higher in control group than in BMSC group by $P<0.001$; IL-4 level was lower in control group than in BMSC group by $P<0.01$. Conclusion: BMSCs are easily to be obtained and cultivated in vitro with strong proliferation ability. Effective EAN model can be established by adoptive immune method. BMSC transplantation could effectively inhibit pathologic condition progression of EAN. BMSC transplantation has inhibitory action on inflammatory cells infiltration in target organ-ischiadic nerve of EAN. BMSC transplantation can effectively inhibit proliferation reaction ability of specific $T$ lymphocytes. BMSC transplantation downregulates serum IFN- $\gamma$, TNF- $\alpha$ level, upregulates protective cytokine IL-4 level.

Keywords: BMSC, EAN, transplant, adoptive immune, cytokine

Cell Research (2008) 18:s138. doi: 10.1038/cr.2008.228; published online 4 August 2008

Correspondence: Yanhong Yin

E-mail: yanhongyin@yahoo.com.cn 